\title{
Modified Modal Strain Energy Method for Analyzing the Dynamic Damping Behavior of Constrained Viscoelastic Structures
}

\author{
Bao Sun ${ }^{1, *}$, Jun Wang ${ }^{2}$, Zhan-long Li ${ }^{2}$, Yuan Qin ${ }^{2}$, Shi-zhong Liu' ${ }^{3}$ and Dick Tomker ${ }^{4}$ \\ ${ }^{1}$ School of Applied Science, Taiyuan University of Science and Technology, Taiyuan 030024, China \\ ${ }^{2}$ School of Mechanical Engineering, Taiyuan University of Science and Technology, Taiyuan 030024, China \\ ${ }^{3}$ School of Transportation and Logistics, Taiyuan University of Science and Technology, Taiyuan 030024, China \\ ${ }^{4}$ University of South Pacific, Port Vila Vanuatu
}

Received 18 April 2017; Accepted 27 October 2017

\begin{abstract}
Modal strain energy (MSE) method is an efficient approximation approach for kinetics parameter calculation of constrained viscoelastic structures. MSE fails to determine the precise dynamic behavior of viscoelastic structures when the stiffness matrix is a complex one. To address this issue, this study proposes a new modified MSE method to calculate the loss factor and natural frequency of a constrained viscoelastic structure on the basis of the correlation analysis of the current modal strain method. The modifying factor changed with the amplitude of the modal order loss factor. A prototype system with four parameters, which was equivalent to a viscoelastic sandwich beam or plate, was used to analyze the error between the new modified method and current methods. The proposed method was applied to a viscoelastic suspension. Results show that the proposed method obtains minimum relative errors of $1.2 \%$ and $2.3 \%$ for structural loss factor and natural frequency as compared with existing methods. This study provides a certain reference for the performance analysis, structural design, and improvement of constrained viscoelastic structures.
\end{abstract}

Keywords: Constrained viscoelastic structure, modal strain energy method, loss factor, natural frequency

\section{Introduction}

Damping reduction has been widely used as a vibration control technology in various fields, such as military, agriculture, aviation, and aerospace. This technology fully utilizes the principle of damping dissipation energy and enhances the kinetics stability of the mechanical system [1]. A constrained viscoelastic structure is composed of lower and upper layers made of elastic material (such as steel and aluminum) with high strength and the middle layer made of viscoelastic material (such as rubber and plastic). This structure can increase loss factor and avoid strengthstiffness loss, thereby leading to efficient vibration damping effect and load function [2].

The vibration damping research on viscoelastic structures involves damping material preparation and damping parameter test kinetics modeling of the composite structure. Kinetics modeling, including loss factor and nature frequency, is a key issue [3]. In recent years, many researchers have investigated the kinetics parameters of damping structures [4-8]. However, viscoelastic dynamic performance is difficult to precisely predict owing to the complex stiffness matrix of viscoelastic structures.

Modal strain energy (MSE) method has been widely applied in many engineering fields to address the abovementioned problem. In this study, a modified MSE method based on previous modifying strategies and basic MSE is proposed by involving a changing factor that varies with loss

*E-mail address: ba0810321@163.com

ISSN: $1791-2377$ @ 2017 Eastern Macedonia and Thrace Institute of Technology. All rights reserved. doi:10.25103/jestr.105.21 factor to precisely evaluate viscoelastic dynamic performance.

Based on the analysis above, a problem using the modified MSE method is examined. The proposed method is applied to an engineering case to validate its accuracy.

\section{State of the art}

MSE is widely used in viscoelastic engineering because of its simple form, relative precision, and less calculation cost compared with traditional methods [9, 10]. The structural loss factor in MSE can be obtained through the ratio of material loss factor to strain energy. Subsequently, complex stiffness is acquired without calculating complex eigenvalues. However, the calculation error in MSE increases with the increase in viscous component, thereby negatively affecting the structural loss factor [10].

Recently, considerable literature reports the development of various related methods, from theoretical analysis to finite element method (FEM) verification, several reliable conclusions on damping loss factor and natural frequency have been provided [11]. Wang [12] adopted a complex eigenvalue method to solve the motion equation with multiple eigenvalues and eigenvectors. This method is extremely complicated and lacks the characteristics of kinetics stress and strain needed for a constrained viscoelastic structure. Jaber [13] first proposed the theoretical method for solving natural frequency and loss factor of complex laminated beams under various constrain conditions. This method employs five hypotheses that depend on the application objects. Alfouneh et al. [14-16] established the super element method for complex laminated 
beams and used complex modulus to describe viscoelastic layer. FEM has also been adopted to calculate natural frequency and modal loss factor. Copetti [17-19] utilized the advantages of FEM and system perturbation method to solve the damping characteristic of viscoelastic structures through ASKA software. Ren et al. [20] used complex modulus to describe the frequency correlation of a laminated viscoelastic material and proposed an iterative method of MSE and eigenvalue to solve the dynamic parameters of viscoelastic laminated structures.

The aforementioned proposed methods present difficulty in dealing with large viscoelastic structures associated with obvious errors. The precision of MSE has been improved using modifying strategies to meet the engineering requirement $[21,22]$. Lv et al. [23] used absolute value method of strain energy (AVMSE) to analyze the damping characteristics of structures. In this method, the modulus of viscoelastic material is replaced by the absolute modulus. Structural loss factor is accurately approximated when large viscoelastic proportion in the complex structure is considered. Reference [24] improved AVMSE and proposed a new MSE method (RMSE). This method needs the strain energy of viscoelastic material to multiply a corrected factor. The comparison showed that RMSE improves accuracy of prediction for the loss factor of viscoelastic material.

Although AVMSE and RMSE are modified MSE methods, their modal correction factors of different orders for viscoelastic material are the same and may cause excessive or insufficient corrections [25]. Considering that different order modals possess significantly different loss factors for viscoelastic material, this study introduces a new correction factor that changes with the loss factor amplitude and proposes a new method called ACMSE.

The remainder of the study is organized as follows. Section 3 proposes ACMSE by analyzing the inner connection between RMSE and AVMSE. Subsequently, modal loss factor and natural frequency are derived using ACMSE. Section 4 analyzes the error distribution on a fourparameter viscoelastic model to validate the effectiveness of ACMSE. Section 5 conducts an engineering application of ACMSE on a viscoelastic suspension to validate the feasibility of ACMSE. Section 6 elaborates the conclusions of the study.

\section{Methodology}

\subsection{Constrained viscoelastic structure}

A complex stiffness matrix is applied on the constrained viscoelastic structure shown in Fig. 1; the motion equation is [25]:

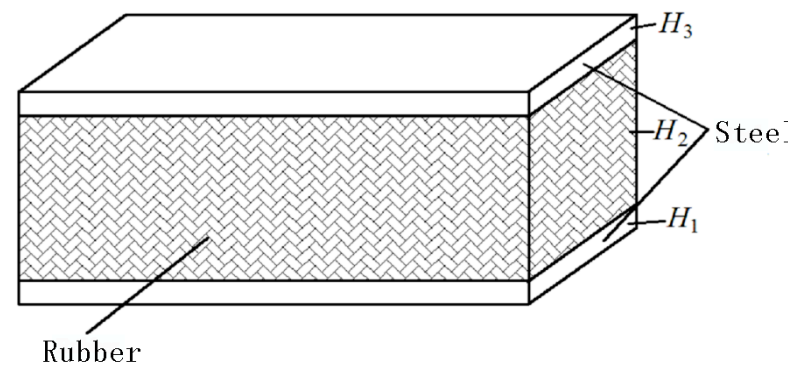

Fig. 1. Constrained viscoelastic structure

$M \ddot{X}+\left(K_{R}+j K_{V I}\right) X=0$
$K_{R}=K_{e R}+K_{V R}$

where $K_{R}$ and $K_{V I}$ are the real and image parts of the complex stiffness matrix, respectively, $K_{e R}$ is the stiffness matrix outside the viscoelastic material, $K_{V R}$ is the stiffness matrix's real part of the viscoelastic material, $M$ is the mass matrix, $X$ is the displacement vector, $j$ is the imaginary unit.

If the constrained viscoelastic structure only contains a type of viscoelastic material and the loss factor of viscoelastic material is $\beta_{V}$, then the following relation can be yield:

$K_{V I}=\beta_{V} K_{V R}$

The solution form of Eq. (1) is:

$X=\Phi_{r}^{*} e^{j \omega_{r}^{*} t}$

Where $\Phi_{r}^{*}$ and $\omega_{r}^{*}$ are the complex eigenvector and complex circle frequency of the $i$-th order, respectively.

According to the analysis of Rao [8]:

$\omega_{r}^{* 2}=\omega_{r}^{2}+j \eta_{r} \omega_{r}^{2}$

Where $\omega_{r}$ is the real part of $\omega_{r}^{*}, \eta_{r}$ is the modal loss factor of the $i$-th order.

When the Rayleigh quotient and complex circle frequency are calculated, the following equation is obtained [8]:

$\omega_{r}^{* T}=\frac{\Phi_{r}^{* T}\left(K_{e R}+K_{V R}+j K_{V I}\right) \Phi_{r}^{*}}{\Phi_{r}^{* T} M \Phi_{r}^{*}}$

\subsection{Brief recall of MSE methods}

\subsubsection{MSE}

In MSE, the real eigenvector $\Phi_{r}$ approximately replaces the complex eigenvector $\Phi_{r}^{*}$, the approximate complex circle frequency $\omega_{r}^{* 2}{ }_{M S E}$ of the $i$-th order is:

$\omega_{r \text { MSE }}^{* 2}=\frac{\Phi_{r}^{T}\left(K_{e R}+K_{V R}\right) \Phi_{r}}{\Phi_{r}^{T} M \Phi_{r}}+j \frac{\Phi_{r}^{T} \beta_{V} K_{V R} \Phi_{r}}{\Phi_{r}^{T} M \Phi_{r}}$

When the real parts of Eqs. (5) and (7) are compared and introduced into Eqs. (2) and (3), the approximate modal loss factor of the $i$-th order is [25]:

$\eta_{r M S E}=\beta_{V} \frac{\Phi_{r}^{T} K_{V R} \Phi_{r}}{\Phi_{r}^{T}\left(K_{V R}+K_{e R}\right) \Phi_{r}}$

\subsubsection{AVMSE}

In AVMSE, the structural complex eigenvector is replaced by the eigenvector calculated by the real part $K_{R}$ of structural stiffness matrix in Eq. (4) without considering the lag influence of viscoelasticity on structural mode shape. The error increases along with the increase of the image 
stiffness in the structural matrix. The influence of image stiffness is considered and modified by taking its absolute value in AVMSE. Real mode $\Phi_{r}$ is obtained by modified stiffness matrix $K_{\alpha}$ and approximately replaces composite structure mode $\Phi_{r}^{*}[26]$.

$K_{\alpha}=\sum_{j=1}^{N E} K_{R_{j}} \sqrt{1+\beta_{j}^{2}}=K_{e R}+\sum_{j=1}^{N V E} K_{V R_{j}} \sqrt{1+\beta_{j}^{2}}$

where $N E$ is the element number, $\beta_{j}$ is the material loss factor of $j$ th element, $N V E$ is the viscoelastic element number, $K_{R_{j}}$ is the stiffness matrix of the $j$-th element, and $K_{V R_{j}}$ is the real part of stiffness matrix of the $j$-th element.

The corresponding eigenvalue problem in this method is:

$\left(-M \bar{\omega}_{r}+K_{\alpha}\right) \Phi_{r}=0$

If $\Phi_{r}$ approximately replaces complex eigenvector $\Phi_{r}^{*}$ and only one type of damping material is present in the structure, then the approximate circle frequency of the $r$-th order in AVMSE is:

$$
\begin{gathered}
\omega_{r \text { AVMSE }}^{* 2}=\frac{\Phi_{r}^{T}\left(K_{e R}+\sqrt{1+\beta_{V}^{2}} K_{V R}\right) \Phi_{r}}{\Phi_{r}^{T} M \Phi_{r}}+ \\
j \frac{\Phi_{r}^{T} \beta_{V} \sqrt{1+\beta_{V}^{2}} K_{V R} \Phi_{r}}{\Phi_{r}^{T} M \Phi_{r}}
\end{gathered}
$$

The approximate mode loss factor $\eta_{\text {rAVMSE }}$ of the $r$-th order is:

$\eta_{r A V M S E}=\beta_{V} \frac{\Phi_{r}^{T} \sqrt{1+\beta_{V}^{2}} K_{V R} \Phi_{r}}{\Phi_{r}^{T}\left(\sqrt{1+\beta_{V}^{2}} K_{V R}+K_{e R}\right) \Phi_{r}}$

\subsubsection{RMSE}

In AVMSE, the calculation of modal loss factor is modified by stiffness matrix and eigenvector and it may cause excessive modification. Rongong [24] proposed a modified method in which Eq. (12) is divided by a modifying factor $\sqrt{1+\beta_{V}^{2}}$ and the approximate mode loss factor $\eta_{r R M S E}$ of the $r$-th order is:

$\eta_{r R M S E}=\beta_{V} \frac{\Phi_{r}^{T} K_{V R} \Phi_{r}}{\Phi_{r}^{T}\left(\sqrt{1+\beta_{V}^{2}} K_{V R}+K_{e R}\right) \Phi_{r}}$

The MSE of viscoelastic material cannot be obtained directly in the finite element analysis in accordance with Eq. (13). $\eta_{r R M S E}$ can be expressed as:

$$
\left\{\begin{aligned}
\eta_{r R M S E} & =\frac{1}{\frac{1}{\beta_{V}}+\alpha_{\text {RMSE }}\left(\frac{1}{\eta_{r A V M S E}}-\frac{1}{\beta_{V}}\right)} \\
\alpha_{\text {RMSE }} & =\sqrt{1+\beta_{V}^{2}}
\end{aligned}\right.
$$

where $\alpha_{R M S E}$ is the modifying factor.

Considering that frequency square is proportional to stored energy [12], the approximate circle frequency $\omega_{r R M S E}$ of the $r$-th order is:

$\omega_{r R M S E}=\lambda_{r A V M S E} \sqrt{\frac{\Phi_{r}^{T}\left(K_{V R}+K_{e R}\right) \Phi_{r}}{\Phi_{r}^{T}\left(\sqrt{1+\beta_{V}^{2}} K_{V R}+K_{e R}\right) \Phi_{r}}}$

Eq. 15 can be rewritten as:

$\omega_{r R M S E}=\lambda_{r A V M S E} \sqrt{\frac{\beta_{V}+\eta_{r A V M S E}\left(1 / \alpha_{R M S E}-1\right)}{\beta_{V}}}$

\subsection{ACMSE}

The modifying factors in AVMSE and RMSE are the same for the viscoelastic composite structure. However, different loss factors theoretically exist for different modal orders, thereby improving the calculation accuracy. Thus, a new modifying factor is proposed in this study on the basis of $\alpha_{r R M S E}$ in RMSE and $0 \leq \eta_{r} \leq \beta_{V}$.

$\left\{\begin{array}{l}\alpha_{r_{A C M S E}}=\sqrt{1+\beta_{V}^{2}-\left(\eta_{r R M S E}+\beta_{V}\right)^{2} / 4} \\ \mu_{r}=1-\frac{\eta_{r R M S E}}{\beta_{V}}\end{array}\right.$

Accordingly, $0 \leq \mu_{r} \leq 1$.

The approximate modal loss factor $\eta_{r A C M S E}$ of the $r$-th order in ACMSE is:

$$
\eta_{r_{A C M S E}}=\frac{1}{\frac{1}{\beta_{V}}+\alpha_{\text {rACMSE }}\left(\frac{1}{\eta_{\text {rAVMSE }}}-\frac{1}{\beta_{V}}\right)}
$$

Similar to the process in RMSE, the approximate circle frequency $\omega_{r A C M S E}$ of the $r$-th order in ACMSE is:

$\omega_{r A C M S E}=\lambda_{r A V M S E} \sqrt{\frac{\beta_{V}+\eta_{r A V M S E}\left(1 / \alpha_{r A C M S E}-1\right)}{\beta_{V}}}$

\section{Result analysis and discussion}

\subsection{Prototype system with four parameters}

The errors of four methods, i.e. MSE, AVMSE, RMSE and ACMSE are analyzed using a prototype system with four parameters, which is equivalent to a viscoelastic sandwich beam (plate), to validate the rationality and accuracy of ACMSE.

Torvik et al. [26]. proposed a prototype system with four parameters (Fig. 2) to analyze MSE.

The complex stiffness of the system can be obtained by the viscoelastic-elastic principle as follows:

$$
K^{*}=\frac{1}{1 / k_{1}+1 /\left(k_{2}+j \beta k_{2}\right)}+k_{3}
$$




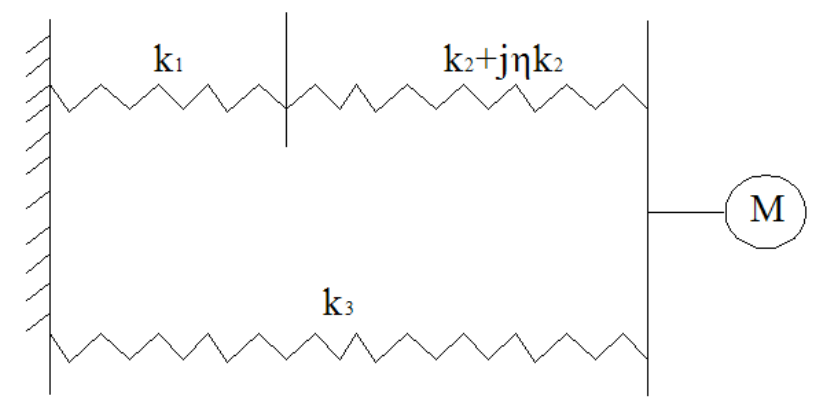

Fig. 2. Prototype system with four parameters

The complex stiffness of the system can be obtained by the viscoelastic-elastic principle as follows:

$$
K^{*}=\frac{1}{1 / k_{1}+1 /\left(k_{2}+j \beta k_{2}\right)}+k_{3}
$$

System loss factor $\eta_{s}$ can be obtained by the ratio of imaginary and real parts of system complex stiffness as follows:

$$
\eta_{s}=\frac{\beta \cdot x}{x^{2}(y+1) \beta^{2}+x^{2}+y x^{2}+x+y+2 x y}
$$

Where $x=k_{2} / k_{1}, y=k_{3} / k_{1}$, and $\beta$ is the loss factor of the complex stiffness spring.

From the system homogeneous solution, the natural frequency $\omega_{s}$ is obtained as:

$\omega_{s}=\operatorname{Re}\left(\sqrt{K^{*} / M}\right)$

Complex stiffness elasticity modulus is replaced by the real part in MSE and by the absolute value in AVSME. The modifying factor is improved in RMSE on the basis of AVMSE, and the structural loss factors and natural circle frequency of these MSE methods are obtained as follows:

$$
\begin{aligned}
& \left\{\begin{array}{l}
\eta_{M S E}=\frac{\beta \cdot x}{(y+x y+x)(1+x)} \\
\omega_{M S E}=\sqrt{\frac{z(y+x y+x)}{1+x}}
\end{array}\right. \\
& \left\{\begin{array}{l}
\eta_{\text {AVMSE }}=\frac{\beta \cdot \alpha \cdot x}{(y+\alpha x y+\alpha x)(1+\alpha x)} \\
\omega_{\text {AVMSE }}=\sqrt{\frac{z(y+\alpha x y+\alpha x)}{1+\alpha x}}
\end{array}\right. \\
& \left\{\begin{array}{l}
\eta_{R M S E}=\frac{1}{\frac{1}{\beta}+\alpha\left(\frac{1}{\eta_{A V M S E}}-\frac{1}{\beta}\right)} \\
\omega_{\text {RMSE }}=\omega_{\text {AVMSE }} \sqrt{\frac{\beta+\eta_{A V M S E}(1 / \alpha-1)}{\beta}}
\end{array}\right.
\end{aligned}
$$

Where $z=k_{1} / M$ and $\alpha=\sqrt{1+\beta^{2}}$.
Similar to the modifying factor improvement functions in RMSE, modifying factor $\alpha^{\prime}$ varies with the system efficient loss factor in ACMSE. The structural loss factor and natural circle frequency are inferred as:

$$
\left\{\begin{array}{l}
\eta_{A C M S E}=\frac{1}{\frac{1}{\beta}+\alpha^{\prime}\left(\frac{1}{\eta_{A V M S E}}-\frac{1}{\beta}\right)} \\
\omega_{A C M S E}=\omega_{A V M S E} \sqrt{\frac{\beta+\eta_{A V M S E}\left(1 / \alpha^{\prime}-1\right)}{\beta}}
\end{array}\right.
$$

where $\alpha^{\prime}=\sqrt{1+\left(1-\eta_{R M S E} / \beta\right) \beta^{2}}$.

From the above-mentioned inference on structural loss factor $\eta$ and natural frequency $\omega$ in these MSE methods, $\eta$ is found related to stiffness ratio $x, y$ and $\beta$. Natural frequency $\omega$ presents a function relationship with stiffness ratio $x, y$, complex spring loss factor $\beta$, and $z$, for convenience of analysis, $z$ is assumed to be constant and negligible [25].

\subsection{Error analysis for loss factor}

The four above-mentioned MSE errors are explored. $x, y$ is in the range of $0.01-100$, indicating four-order change in stiffness ratio; $\beta$ is in the range of $0-2$, representing the loss factors of common viscoelastic material. The relative error curves of structural loss factor and natural frequency in MSE, AVMSE, RMSE, and ACMSE are shown in Figs. 3 and 4.

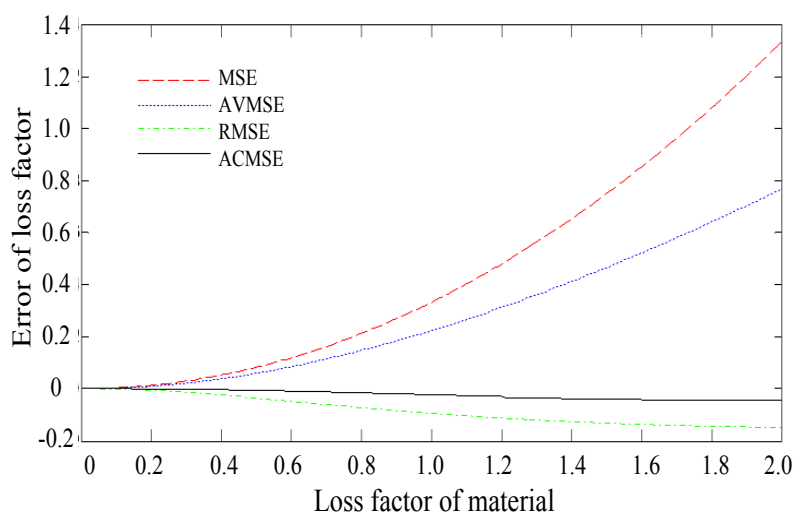

(a) $k_{2} / k_{1}=1, k_{3} / k_{1}=1, \beta \in[0,2]$

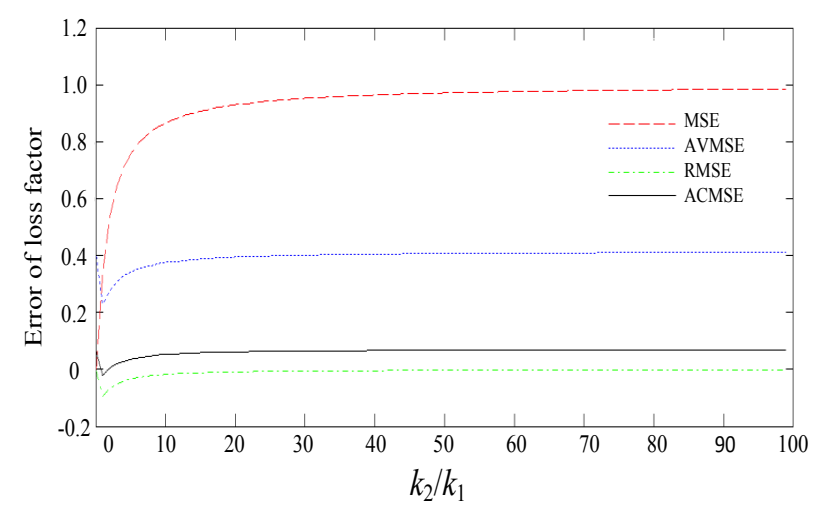

(b) $\beta=1, k_{3} / k_{1}=1, k_{2} / k_{1} \in[0.01,100]$ 


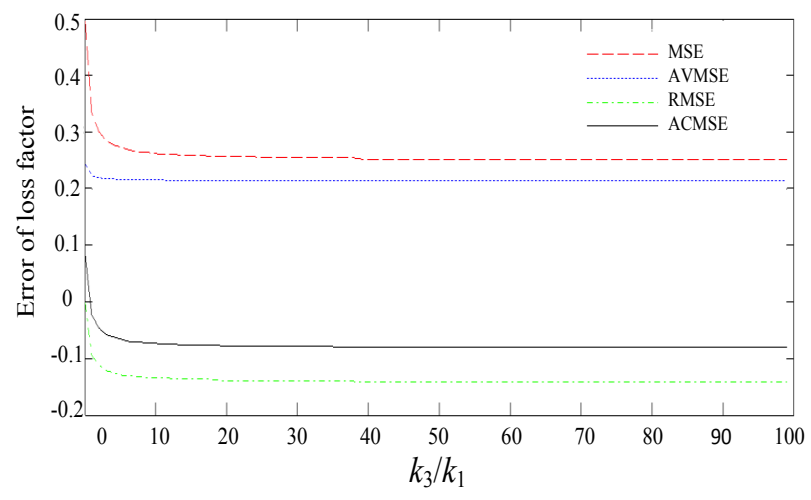

(c) $\beta=1, k_{2} / k_{1}=1, k_{3} / k_{1} \in[0.01,100]$

Fig. 3. Relative error calculation for various loss factors

The following conclusions can be obtained from the analysis above.

When stiffness ratio $x, y$ is invariable, the errors of structural loss factor in the four methods increase with the increase in material loss factor. The error in MSE is particularly obvious. Thus, the method needs to be improved.

Compared with AVMSE and RMSE, ACMSE obtains minimum structural loss factor error with a lower bound.

When $\beta$ and stiffness ratio $k_{3} / k_{1}, k_{2} / k_{1}$ are invariable, the structural loss factor error in ACMSE is the minimum. With the increase in stiffness ratio, the error stabilizes.

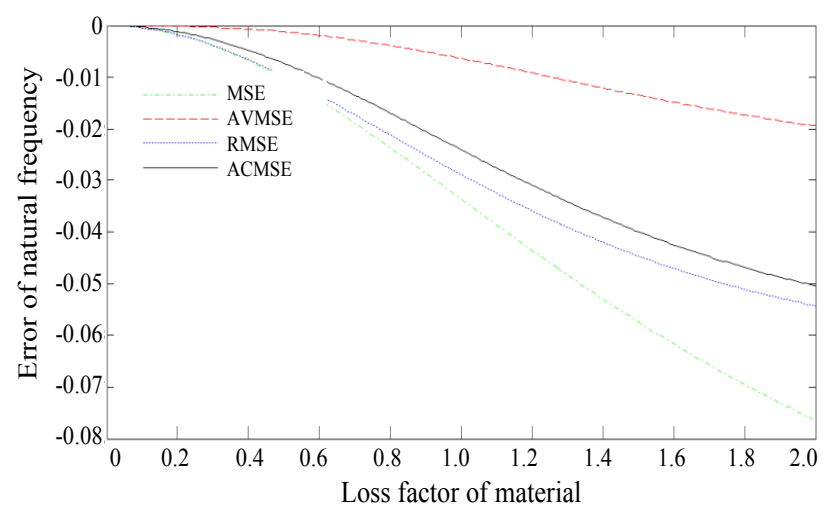

(a) $k_{2} / k_{1}=1, k_{3} / k_{1}=1, \beta \in[0,2]$

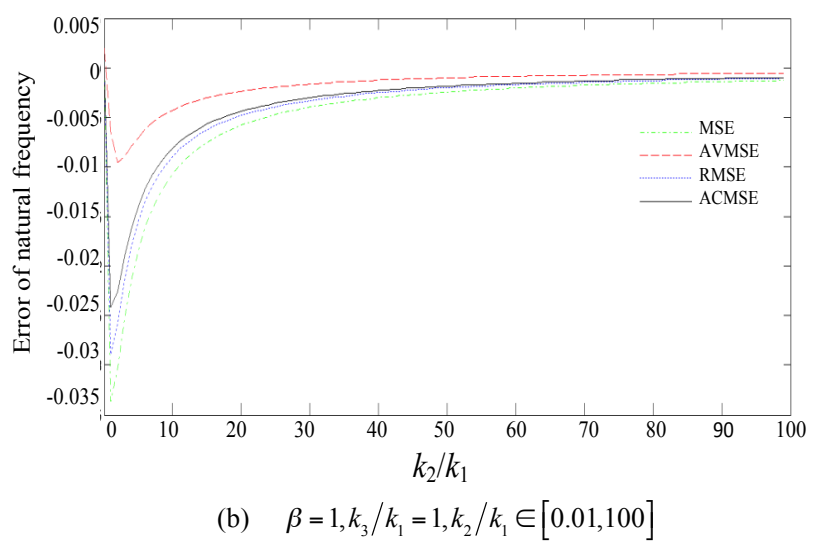

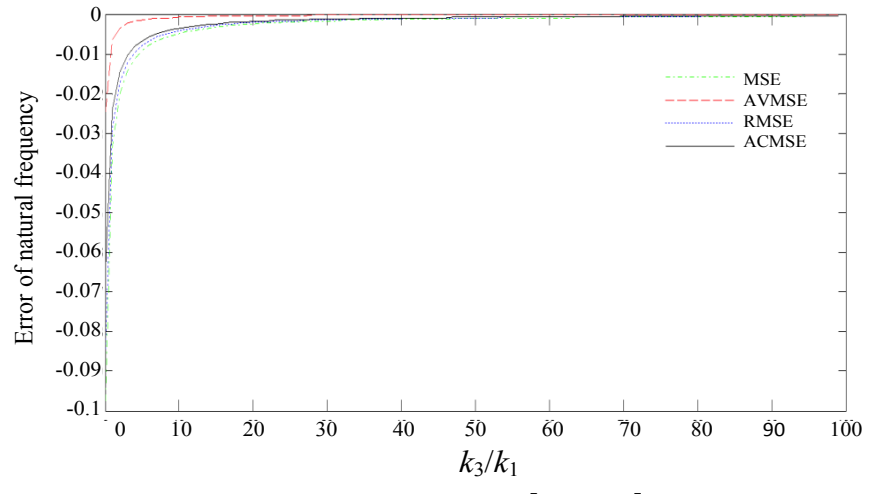

(c) $\beta=1, k_{2} / k_{1}=1, k_{3} / k_{1} \in[0.01,100]$

Fig. 4. Relative error calculation for natural frequency by various MSE

\subsection{Error analysis for natural frequency}

Fig. 4 shows the relative error calculation for natural frequency by various MSE methods.

From the relative error curves for natural frequency by various MSE methods, the following conclusions are drawn.

When stiffness ratio $x, y$ is invariable, the errors of structural loss factor in the four methods increase with the increase in material loss factor. The error in MSE is the maximum and increases rapidly.

The natural frequency error in AVMSE is the minimum. The error in ACMSE is only slightly larger than that in AVMSE whereas smaller than the errors in MSE and RMSE.

When material loss factor $\beta$ and stiffness ratio are invariable and $k_{2} / k_{1}$ is in a very small area, the errors of the four methods increase with $k_{2} / k_{1}$. The error in ACMSE is larger than that in AVMSE only. With the increase in $k_{2} / k_{1}$, the errors in these methods decrease and stabilize.

When material loss factor $\beta$ and stiffness ratio are invariable, the errors in these methods decrease with the increase in $k_{3} / k_{1}$. The error in AVMSE is the smallest, and that of ACMSE is smaller than the errors in MSE and RMSE.

With the increase in $k_{3} / k_{1}$, the errors in the four methods are very close and stable in general.

In summary, the proposed ACMSE can more efficiently calculate structural loss factor and natural frequency among all the compared methods.

\subsection{Example verification}

A constrained viscoelastic structure is used to validate ACMSE. The structure is mounted on a crawler bulldozer to absorb vibration and shock. The structure uses damping technology and thus possesses good buffer damping performance [27].

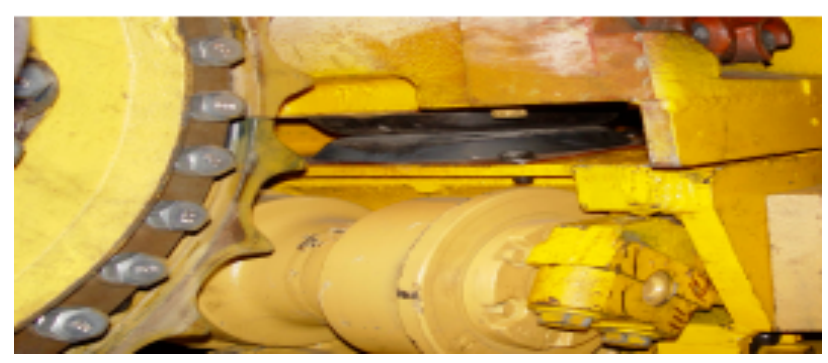

Fig. 5. Picture of viscoelastic suspension 
The loss factor and natural frequency of a viscoelastic suspension installed in a bulldozer with 410 horsepower (Fig. 5) are calculated using the four MSE methods. The precision of ACMSE compared with that of other MSE methods is validated using ANSYS software by FEM.
The mechanical behavior of steel and rubber is simulated by $3 \mathrm{D}$ element Solid 185 . The steel and rubber materials are regarded as isotropic. The model and physical parameters used in the FEM analysis are shown in Tab. 1.

Table 1. Main geometric and physical parameters of viscoelastic suspension's rubber pad

\begin{tabular}{|c|c|c|c|c|c|}
\hline Geometrical parameters of rubber & Parameter & Structural layer & Density $\rho$ & Elasticity modulus, shear & Poisson's \\
\hline base diameter $D / \mathrm{mm}$ & 305 & \multirow{7}{*}{$\begin{array}{l}\text { Restraint Layer } \\
\text { Damping Layer } \\
\text { Basement Layer }\end{array}$} & \multirow{7}{*}{$\begin{array}{l}7800 \\
1130 \\
7800\end{array}$} & \multirow{7}{*}{$\begin{array}{l}2.100 \times 1011 \\
0.896 \times 106 \\
2.100 \times 1011\end{array}$} & \multirow{7}{*}{$\begin{array}{l}0.300 \\
0.499 \\
0.300\end{array}$} \\
\hline Total height $h_{t} / \mathrm{mm}$ & 45 & & & & \\
\hline ceiling height $h_{u} / \mathrm{mm}$ & 10.4 & & & & \\
\hline central height $h_{m} / \mathrm{mm}$ & 30.6 & & & & \\
\hline Bottom height $h_{b} / \mathrm{mm}$ & 4.0 & & & & \\
\hline Central support angle $\theta /\left({ }^{\circ}\right)$ & 55 & & & & \\
\hline Total radius of circular arct $R / \mathrm{mm}$ & 950 & & & & \\
\hline
\end{tabular}

The loss factor entity model of the damping structure in Fig. 5, FEM, stresses, and strain image under $3 \mathrm{~Hz}$ of a typical working condition are shown in Figs. 6, 7, 8, and 9, respectively.

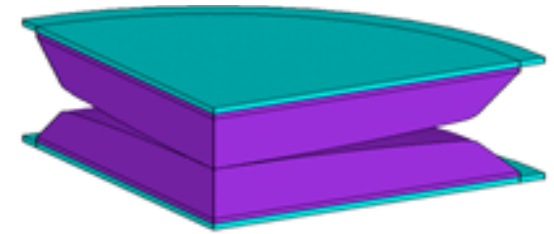

Fig. 6. One-fourth symmetric 3D model of viscoelastic suspension

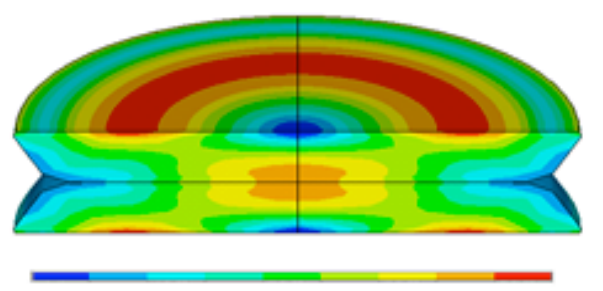

Fig. 8. Stress contour picture for viscoelastic damping structure at $3 \mathrm{~Hz}$
The results of loss factor and natural frequency of the damping structure analyzed by ANSYS using ACMSE and three other MSE methods are shown in Table 2.

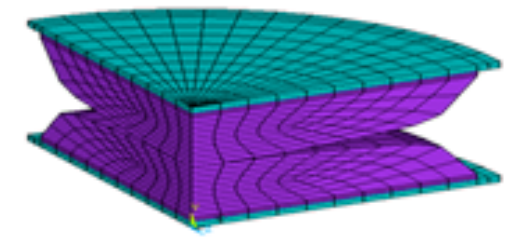

Fig. 7. One-fourth symmetric 3D FEM model of viscoelastic suspension

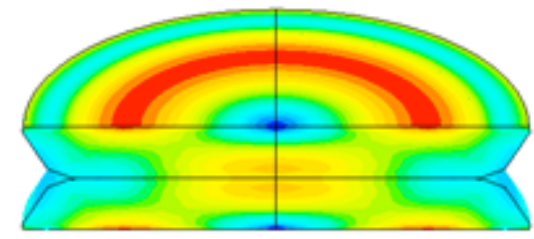

Fig. 9. Strain contour picture for viscoelastic damping structure at $3 \mathrm{~Hz}$

Table 2. Loss factor and natural frequency calculation of MSE methods

\begin{tabular}{|c|c|c|c|c|c|c|c|c|c|c|}
\hline \multirow{2}{*}{ Modal } & \multicolumn{2}{|c|}{ MSE result } & \multicolumn{2}{|c|}{ AVMSE result } & \multicolumn{2}{|c|}{ RMSE result } & \multicolumn{2}{|c|}{ ACMSE result } & \multicolumn{2}{|c|}{ ANSYS result } \\
\hline & Loss factor & Natural & Loss factor & Natural & Loss factor & Natural & Loss factor & Natural & Loss factor & Natural \\
\hline 1 & 0.198 & 267.35 & 0.274 & 283.25 & 0.275 & 282.21 & 0.296 & 283.15 & 0.298 & 285.35 \\
\hline 2 & 0.203 & 267.83 & 0.269 & 283.69 & 0.279 & 282.53 & 0.306 & 28 & 0.314 & 83 \\
\hline 3 & 0.221 & 271.96 & 0.280 & 284.36 & 0.281 & 282.94 & 0.324 & 284.06 & 0.325 & 286.46 \\
\hline 4 & 0.216 & 273.52 & 0.291 & 284.68 & 0.289 & 283.31 & 0.318 & 284.18 & 0.319 & 286.52 \\
\hline 5 & 0.231 & 275.76 & 0.289 & 285.26 & 0.294 & 283.25 & 0.329 & 285.23 & 0.333 & 291.96 \\
\hline
\end{tabular}

The loss factor error in ACMSE is the smallest compared with the errors in ACMSE and MSE. The natural frequency errors in the three other MSE methods are smaller than the error in MSE, and the error in ACMSE is only smaller than that in AVMSE. This conclusion is consistent with the further error analysis and proves the rationality and accuracy of ACMSE. Therefore, the proposed method can meet the requirement of a few engineering designs.

\section{Conclusions}

To evaluate the dynamic behavior of viscoelastic structure more precisely, this study analyzed the characteristics and relations of MSE, AVMSE, RMSE, and proposed an ACMSE with loss factor varies. The following conclusions could be drawn..
(1) The modifying factor changes with the corresponding modal loss factor; thus, the calculation accuracy of ACMSE for viscoelastic damping features is better than the accuracies of MSE, AVMSE, and RMSE.

(2) The error analysis shows that the natural frequency and modal loss factor errors are $1.2 \%$ and $2.3 \%$, respectively, for ACMSE. This finding also proves the accuracy of ACMSE.

(3) ACMSE can be applied not only to the kinetics analysis of viscoelastic constrained damping structure with two elastic layers but also to complex constrained damping structures with multiple layers.

This research proposed a new MSE method namely ACMSE for analyzing dynamic response of multiple-layer constrained viscoealastic damping structure. On the other hand, it provides a certain reference for the performance analysis, structural design, and improvement of constrained 
viscoelastic structures. The material loss factor presents a certain non-linear variation for large strain condition. Therefore, the future study will comprehensively explore the application of MSE into the non-linear kinetics parameter analysis of damping structures with multiple constraints.

\section{Acknowledgments}

This work was supported by Taiyuan University of Science and Technology Fund for Doctor (20162005, 20162035,20162010).

Access article distributed under the terms of the Creative Commons Attribution License

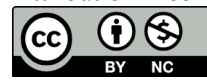

\section{References}

1. Tian $\mathrm{S}, \mathrm{Xu} Z \mathrm{Z}, \mathrm{Wu} \mathrm{Q}$, et al., "Dimensionless Analysis of Segmented Constrained Layer Damping Treatments with Modal Strain Energy Method". Shock and Vibration, 2016(1),2016,pp.1-16.

2. Shen Yanli, Yang Qingshan, Tian Yuji., "Study of accuracy and applicability of modal strain enegy method". Engineering Mechanics, 25(6), 2008,pp.18-21.

3. Gui Hongbin, Zhao Deyou, Zheng Yunlong., "A review finite element method analyzing dynamic problem of structure with viscoelastic damped layer". Jounal of Vibration and Shock, 20(1), 2001,pp.44-47.

4. He Y C, Chen W L, Sun S W, et al., "Finite Element Research on Damping of Viscoelastic Free Layer Damping Sheet”. Applied Mechanics \& Materials, 472(23), 2014,pp.56-61.

5. Chen W, Liu S., "Topology optimization of microstructures of viscoelastic damping materials for a prescribed shear modulus". Structural \& Multidisciplinary Optimization, 50(2), 2014,pp.287296.

6. Zghal S, Bouazizi M L, Bouhaddi N, et al., "Model reduction methods for viscoelastic sandwich structures in frequency and time domains". Finite Elements in Analysis \& Design, 93(2), 2015,pp.12-29.

7. Tirella A, Mattei G, Ahluwalia A., "Strain rate viscoelastic analysis of soft and highly hydrated biomaterials". Journal of Biomedical Materials Research Part A, 102(10), 2014,pp.3352-3360.

8. Seyedpoor S M., "A two stage method for structural damage detection using a modal strain energy based index and particle swarm optimization". International Journal of Non-Linear Mechanics, 47(1), 2012,pp.1-8.

9. Sun Wei, Hao Chunlei, Zhang Honghao., "Modified modal strain energy method used for predicting damping characteristics of hard-coating thin plate". Journal of Northeastern University, 35(4), 2014,pp.551-554.

10. Huang J, Zhou Z, Zhang L, et al., "Strain Modal Analysis of Small and Light Pipes Using Distributed Fibre Bragg Grating Sensors". Sensors, 16(10), 2016,pp.1583-1589.

11. Ren D X, Zhang D Y, He Y F, et al., "Vibration response investigation on structures with particle metal rubber damper fillings". Tuijin Jishu/journal of Propulsion Technology, 36(1), 2015, pp.124-129.

12. Wang Zhengxin, Dai huijun., "An optimized computation for damping elastic plank structure". Noise and Vibration Control, 18(6), 2000,pp.21-30.

13. Jaber M, Schneeweiss H, Bos J, et al., "Vibrational energy flow in carbon composite structures". Meat Science, 84(1),2014,pp.180185 .
14. Khusnutdinova, Ilvina Gamirovna, Bashirov M G., "The Use of Electromagnetic-Acoustic Method for Estimating the Stress-Strain State of the Metallic Elements of Power Equipment". Key Engineering Materials, 743(45), 2017,pp.463-467.

15. Alfouneh M, Tong L., "Maximizing modal damping in layered structures via multi-objective topology optimization". Engineering Structures, 132, 2017,pp.637-647.

16. Moradipour P, Chan T H T, Gallage C., "Benchmark Studies for Bridge Health Monitoring Using an Improved Modal Strain Energy Method". Procedia Engineering, 188(45), 2017,pp.194-200.

17. Copetti M I M, Fernández J R, Masid M., "Numerical analysis of a viscoelastic mixture problem". International Journal of Solids and Structures, 80(2), 2016,pp.393-404.

18. Amabili M., "Nonlinear vibrations of viscoelastic rectangular plates". Journal of Sound and Vibration, 362(34),2015, pp.142156.

19. Lewandowski R, Łasecka-Plura M., "Design sensitivity analysis of structures with viscoelastic dampers". Computers and Structures, 164(1), 2016,pp.95-107.

20. Ren Zhigang, Lu Zhean., "Calculation of frequency and loss factor for composite sandwich structures". Earthquake Engineering and Engineering Vibration,24(2), 2004,pp.101-106.

21. Guo Zhongze, Luo Jingrun, Chen Yuze., "An improved method of predicting the modal loss factors of constrained damping structure". Acta Armamentarii,27(6), 2006,pp.1064-1067.

22. Ding Z, Li L, Hu Y., "A free interface component mode synthesis method for viscoelastically damped systems". Journal of Sound and Vibration, 365(25), 2016,pp.119-215.

23. Lv Gang, Lu Feng, Zhang Jinghui., "Absolute value modal strain energy method for viscoelastic damping control of truss structure". Journal of Astronautics, 20(2),1999,pp.112-116.

24. Rongong J A., "Reducing Vibration levels using 'Smart Joint' concepts". In: Proceedings of IMSA 25th Noise and Vibration Engineering, Belgium, German:Leuven ,2000,pp.153-162.

25. Li Shiqi, Zhang Zhenli, Zhu Wenge., "A new modal strain energy method for analyzing dynamic parameters of viscoelastic structures". Noise and Vibration Control, 12(18), 2011,pp.47-52.

26. Torvik P J, Runyon B., "Modifications to the method of modal strain energy for improved estimates of loss factors for damped structures". Shock and Vibration, 14(5),2007,pp.339-353.

27. Sun Dagang, Song Yong, Lin Muyi., "Modeling of dynamic contact finite element method for damping buffer components mounted on viscoelastic suspensions". Transactions of the Chinese Society of Agricultural Engineering,24(1),2008,pp.24-28. 